\title{
RoboCup Rescue Robot and Simulation Leagues
}

\author{
H. Levent Akin, Nobuhiro Ito, Adam Jacoff, \\ Alexander Kleiner, Johannes Pellenz, Arnoud Visser
}

The RoboCup Rescue Robot and Simulation competitions have been held since 2000. The experience gained during these competitions has increased the maturity level of the field, which allowed deploying robots after real disasters (for example, the Fukushima Daiichi nuclear disaster). This article provides an overview of these competitions and highlights the state of the art and the lessons learned.

\begin{abstract}
$\Lambda$ fter the Great Hanshin Earthquake of 1995 in Kobe, the Japanese government decided to promote research related to the problems encountered during large-scale urban disasters. A major outcome of this initiative was the RoboCup Rescue competitions. In this article, we introduce the RoboCup Rescue leagues, namely the Rescue Robot League (RRL) and the Rescue Simulation League (RSL) (Tadokoro et al. 2000; Kitano and Tadokoro 2001).

Disaster mitigation is an important social issue involving large numbers of heterogeneous agents acting in hostile environments. The associated urban search and rescue (USAR) scenarios have great potential for inspiring and driving research in multiagent and multirobot systems. Since the circumstances during real USAR missions are extraordinarily challenging (Murphy et al. 2008), benchmarks based on them, such as the RoboCup Rescue competitions, are ideal for assessing the capabilities of intelligent robots. Robots that can navigate through affected areas after a disaster most likely will also be capable of negotiating the very same environment under normal circumstances. If robots are able to recognize humans entombed under piles of rubble of collapsed buildings, they will also be able to recognize them within their natural environment.
\end{abstract}


The goal of the RoboCup Rescue competitions is to compare the performance of different algorithms for coordinating and controlling teams of either robots or agents performing disaster mitigation in a simulated yet realistic environment. By their nature, the USAR scenarios demand solutions for the coordination of large and distributed teams of heterogeneous robots.

The competitions are held annually as a part of the RoboCup World Championships. In addition, regional competitions are held in RoboCup Opens such as the ones in the United States, Germany, the Netherlands, Japan, Iran, Thailand, and China. The RoboCup Opens are organized by the national committees, whereas the international competitions are organized by league organization committees. The competition rules are determined by the technical committees, whose members are elected annually.

Due to the complexity of the problems addressed in the competitions, the barrier for the entry of new teams is high. For this reason, the leagues have recently introduced several measures for facilitating software reuse among the teams.

In the following sections we describe the competitions in RRL and RSL.

\section{Rescue Robot League}

The Rescue Robot competitions started in 2000 as a part of the AAAI Mobile Robot Competition and Exhibition, and was later integrated into RoboCup in 2001. In 2012, approximately 100 teams competed in the regional opens; 11 teams from seven nations qualified for the world championship in RoboCup 2012 held in Mexico City.

The objective of this league is to promote the development of intelligent, highly mobile, dexterous robots that can improve the safety and effectiveness of emergency responders performing hazardous tasks. Annual competitions and subsequent field exercises with emergency responders are used to increase awareness about the challenges involved in deploying robots for emergency response applications. They are also used to provide objective performance evaluations based on DHS-NIST-ASTM international standard test methods for response robots, introduce best-in-class implementations to emergency responders within their own training facilities, and support ASTM International Standards Committee on Homeland Security Applications (E54.08.01).

The main task of the competition is to find simulated victims emitting several signs of life in a maze with different levels of harsh terrain and to indicate the location of the victims and of automatically detected landmarks on a map generated by the robot online. The signs of life can be the visual appearance and movements of the victims, simulated body heat, $\mathrm{CO}_{2}$ levels, and audio signals. The harsh terrain is built of different ramps, stairs, and so-called step fields, which consist of a normed pattern of wooden blocks resembling the structure of a rubble pile (see figure 1).

Both remote-controlled and autonomous robots are allowed in the competitions to foster the improvement of the mobility of the robots, human-robot interaction, and the development of autonomous agents that are capable of handling such unstructured environments. To highlight the achievements in these categories special awards are given for Best-in-Class Autonomy, Best-in-Class Mobility, and Best-in-Class Manipulation.

Due to the use of standardized test elements, the progress of the league over the years can readily be seen. When the random step fields were introduced in 2005, none of the robots were able to cross them in a reliable manner. Today, driving through the step fields is the state of the art for the remote-controlled robots. During the same time, fully autonomous robots had to learn handling uneven terrains since their section of the arena was no longer flat, but increasingly filled with continuous or crossed ramps. Such a challenging environment increases the complexity of path planning and requires an active sensing approach with stabilized distance and victim sensors. Nevertheless, the quality of the generated maps has increased over the years, and all of the competing teams can now map the whole arena. An example of a very precise map is given in figure 2 . This map shows both the obstacles in the arena and also the victim locations and other landmarks found autonomously by the on-board vision system. These maps are scored based on the completeness of the exploration and accuracy.

The scientific output of the league over the years include online simultaneous localization and mapping (SLAM) solutions (Kohlbrecher et al. 2011; Pellenz and Paulus 2009; Kleiner and Dornhege 2009) and solutions for efficient exploration strategies (Wirth and Pellenz 2007). The performance of these approaches was demonstrated and tested in practice during several RoboCup competitions. The mapping system introduced by Kleiner et al. (2005) has also been integrated into a commercial robot platform for bomb disposal by the German company telerob $\mathrm{GmbH}$ and has also been nominated for the EU technology transfer award for 2011. The robot Quince developed by Japanese universities and evaluated in the RRL in various iterations was finally deployed at the Fukushima Daiichi nuclear power plant in the aftermath of the massive earthquake and tsunami that hit eastern Japan in 2011. It was used for inspection missions in highly contaminated areas (Nagatani et al. 2011).

The league has recently established an open source standard software solution based on Robot 


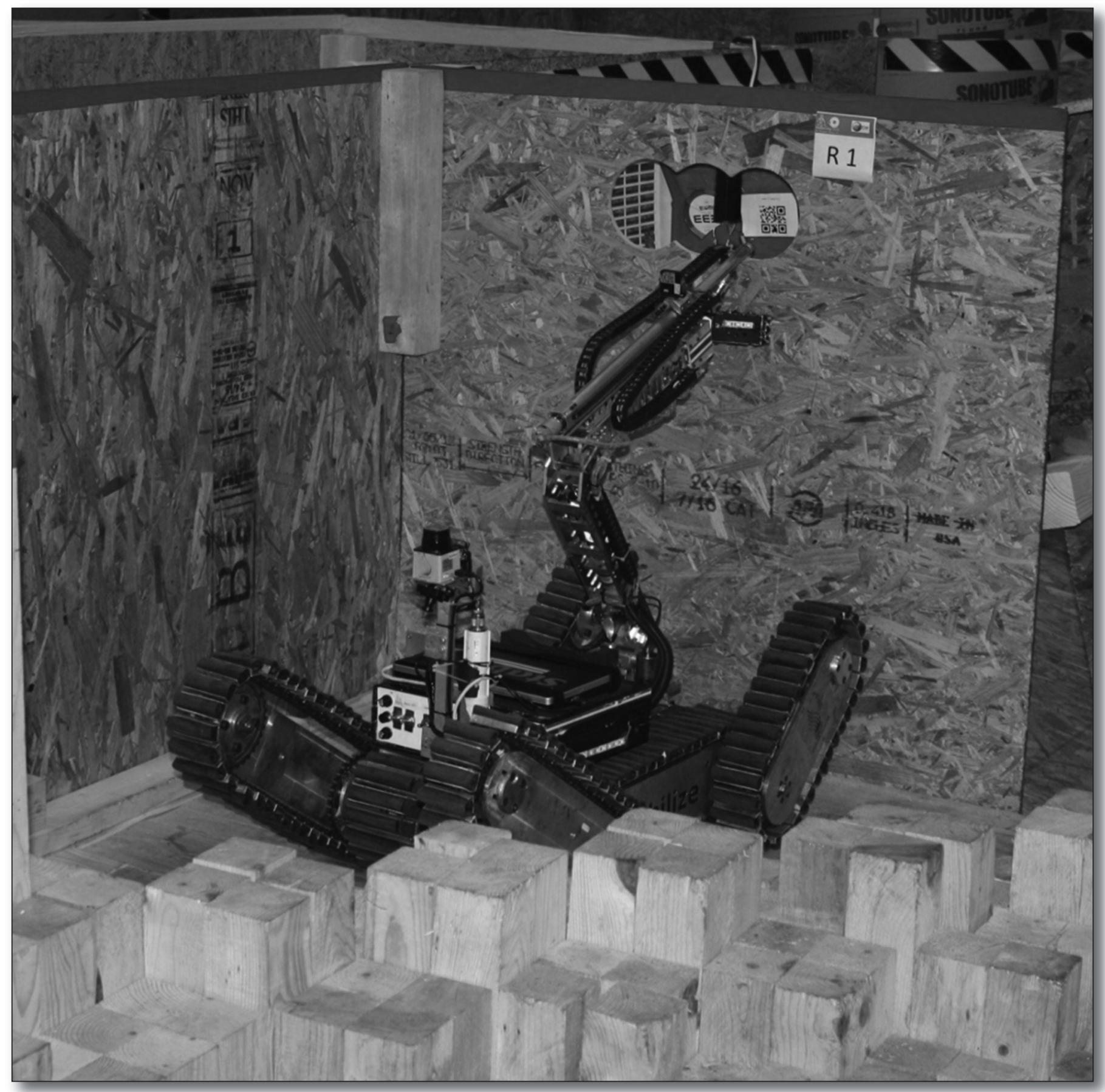

Figure 1. Rescue Robot of Team Stabilize from Thailand Inspects a Simulated Victim near a Step Field at RoboCup 2012.

Operating System (ROS) that makes the best algorithms broadly reusable, thus enabling the new teams to reach quickly the world class performance level. ${ }^{1}$

\section{Rescue Simulation League}

This league aims to develop realistic simulation environments for benchmarking intelligent soft- ware agents and robots that are expected to make rational decisions autonomously in a disaster response scenario. RSL has two major competitions, namely the Agent and the Virtual Robot competitions. There are described in the subsequent sections.

\section{Agent Competition}

The rescue agent competition aims to simulate 


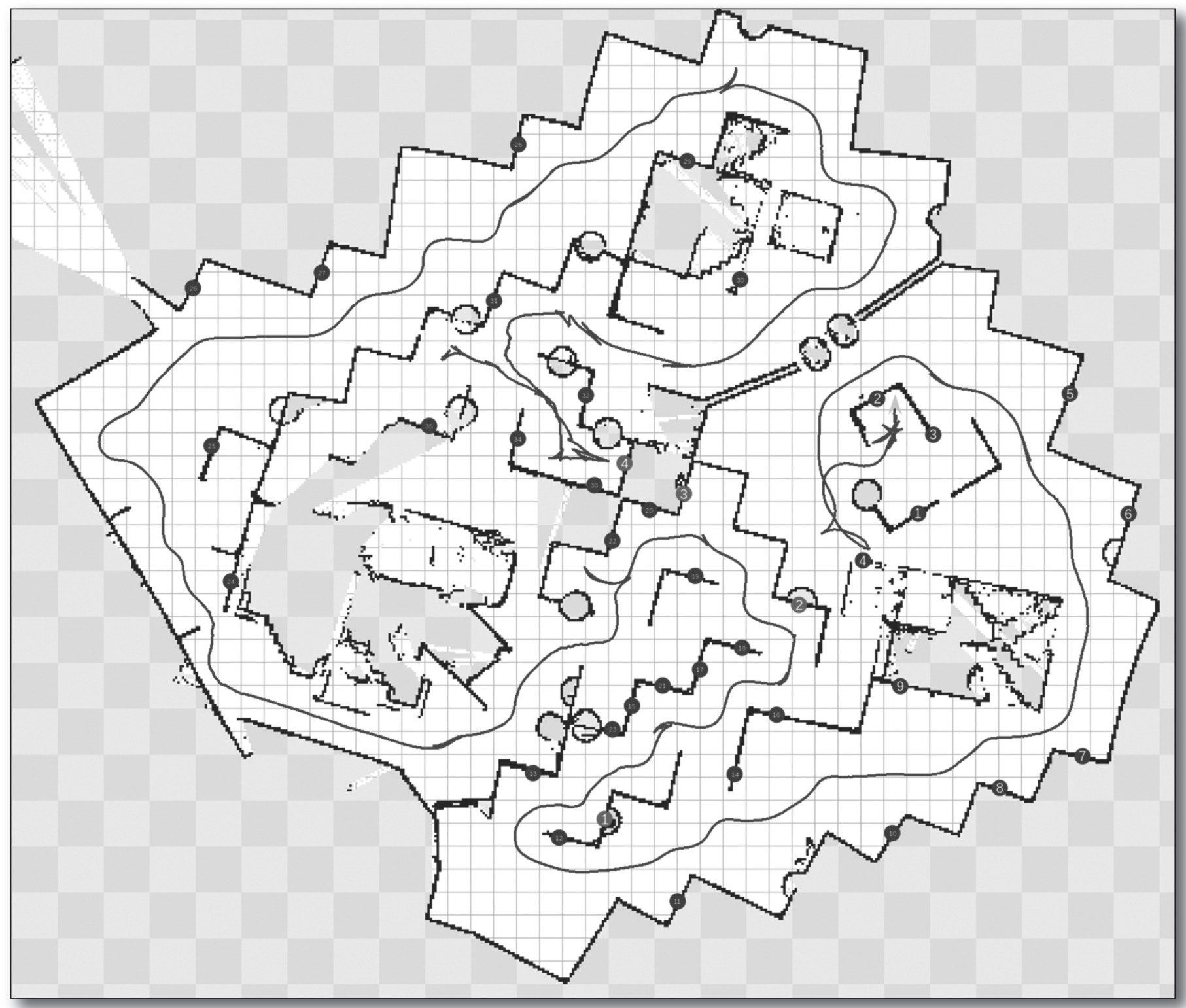

Figure 2. Map Generated by an Autonomous Robot of Team Hector from TU Darmstadt (RoboCup RRL Final 2012).

large-scale disasters such as earthquakes and explore new ways of autonomous coordination of heterogeneous rescue teams under adverse conditions (Kitano et al. 1999; Tadokoro et al. 2000). This competition was first demonstrated during RoboCup 2000 and was later launched as an official competition in 2001.

About 25 university teams participate in the agent competition annually. These teams have their background mainly from AI and robotics and come from different countries such as the United States, Japan, Germany, England, Portugal, Turkey, Iran, and China. Table 1 summarizes the number of teams that participated during the last years.

The competition is based on a complex simula-

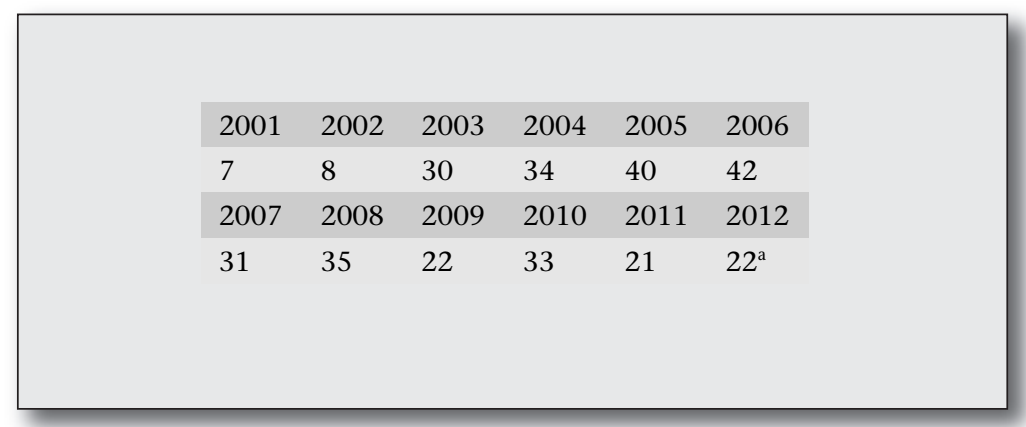

Table 1. The Numbers of Teams That Participated in the Agent Competition during the Last Few Years.

a. Includes six teams of the RMas-Bench challenge. 


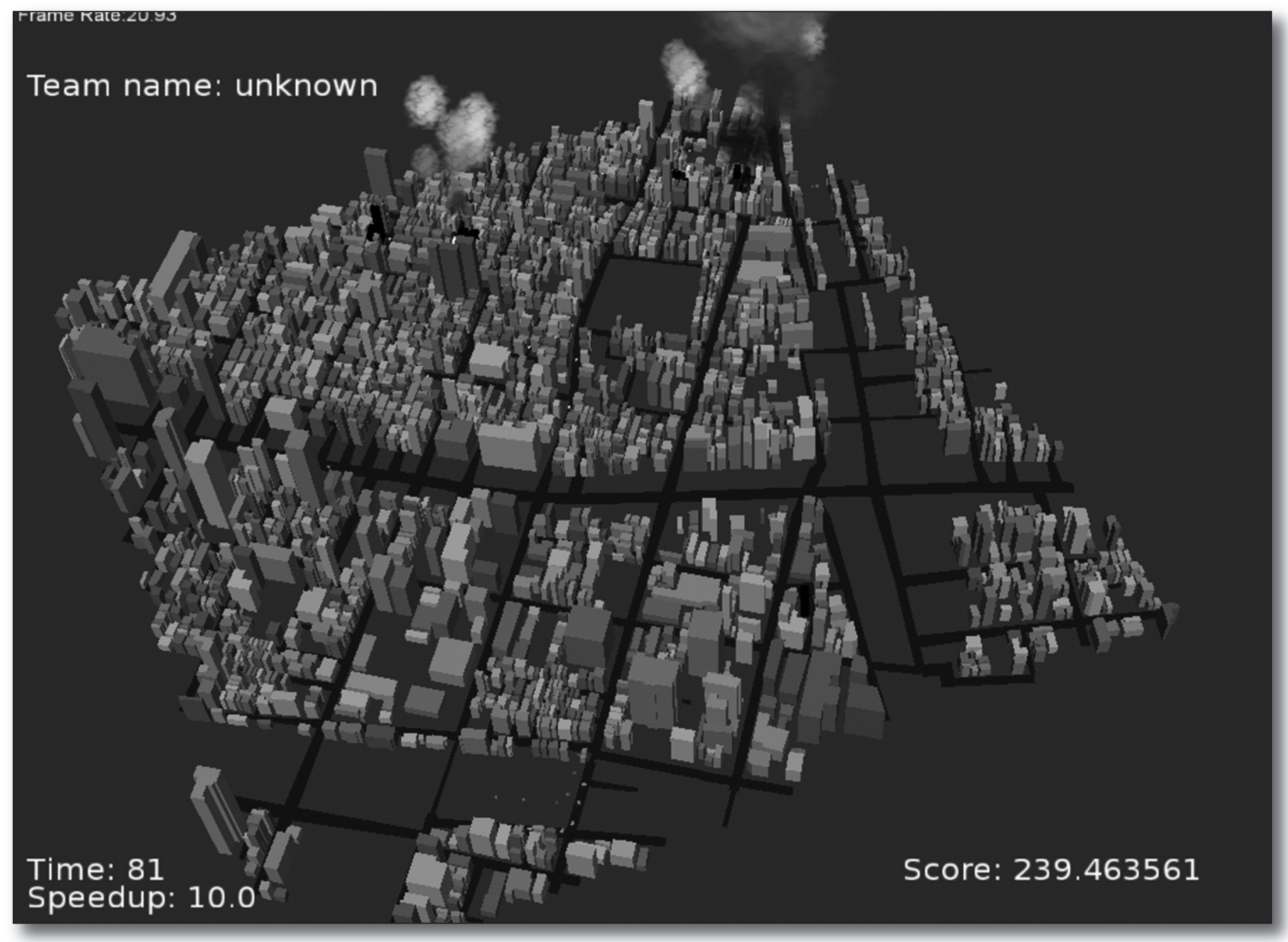

Figure 3. A Typical Scenario Involving the Simulation of Fires and Building Collapse on a Model of the City of Kobe in Japan.

tion platform representing a city after an earthquake (figure 3). Due to the variations among potential disaster scenarios, typically rather than a single agent type, strongly diverse teams of agents are required. In the competitions, teams of fire brigade, police, and ambulance agents try to extinguish fires and rescue victims in the collapsed buildings. The scoring is based on the number of victims saved on time and the number of remaining buildings with various levels of fire damage. The urban areas considered in the competitions typically contain up to 5000 buildings and each of the fire brigade, police, and ambulance teams may consist of at most 30 agents. There may be up to 200 civilians that need to be rescued.

The overall goal of developing robust software systems that are capable of efficiently coordinating large agent teams for USAR raises several research challenges such as the exploration of large-scale environments in order to search for survivors, the scheduling and planning of time-critical rescue missions, coalition formation among agents, and the assignment of agents and coalitions to dynamically changing tasks. In the target domain, these issue are even more challenging due to the restricted communication bandwidth. Moreover, the simulated environment is highly dynamic and only partially observable by the agents. Under these circumstances, the agents have to plan and decide their actions asynchronously in real time while taking into account the long-term effects of their actions.

Over the years the winning entries in the competition showed a strong focus on highly optimized computations for multiagent planning and model-based prediction of the outcome of the ongoing incidents. Several techniques for multiagent strategy planning and team coordination in dynamic domains have also been developed based on the rescue simulator. Task allocation problems inherently found in this domain were first described by Nair, Ito, Tambe, and Marsella (2002). 
The solutions developed for the distributed constraint-optimization problems (DCOPs) encountered during the simulations were evaluated in the paper by Ferreira, Dos Santos, Bazzan, Epstein, and Waskow (2010). The coalition formation with spatial and temporal constraints (CFST) model and the state-of-the-art DCOP algorithms used for solving CFSTs were given in Ramchurn, Farinelli, Macarthur, and Jennings (2010). The problem of scheduling rescue missions were identified and described together with a real-time executable solution based on genetic algorithms by Kleiner, Brenner, Bräuer, Dornhege, Göbelbecker, Luber, Prediger, Stückler, and Nebel (2005).

Furthermore, there has been substantial work on building information infrastructure and decision support systems for enabling incident commanders to efficiently coordinate rescue teams in the field. For example, Schurr and Tambe (2008) introduced a system based on software developed in the rescue competitions for the training and support of incident commanders in Los Angeles.

RSL aims to ease the entry for newcomer teams. However, the rich set of problems that need to be addressed by the agent teams competing in the competition and the degree of sophistication of current successful solutions makes this a nontrivial task. RMas-Bench ${ }^{2}$ is a challenge recently introduced for this purpose. Certain aspects such as task allocation, team formation, and route planning are extracted from the entire problem addressed by the agents and are presented as subproblems in standalone scenarios with an abstract interface. Consequently, the participating teams are able to focus more on the topics relevant to their own research rather than dealing with all of the low-level issues. Currently, RMas-Bench has a generic API for DCOP algorithms together with reference implementations of state-of-the-art solvers, such as DSA (Fitzpatrick and Meetrens 2003) and MaxSum (Farinelli et al. 2008).

In order to facilitate code sharing among the participating teams, a novel protocol for interagent communication is currently being developed in RSL to be integrated with the official release next year. This new addition will increase the modularity of agent solutions and will also allow combination of different agent solutions within a single team.

\section{Virtual Robot Competition}

RoboCup Virtual Robot competitions are being held since 2006. The competition attracts mainly academic teams from universities often with experience in the RoboCup Rescue Robot League. The number of participating teams is typically eight (table 2), but this is partly due to the strict qualification rules of this competition.

The important research issues related to the

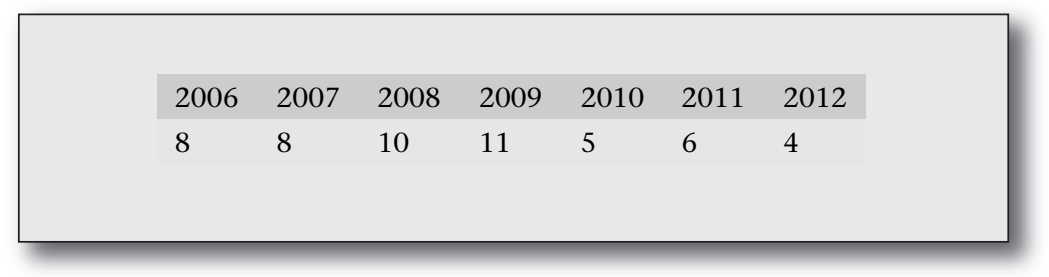

Table 2. The Number of Teams that

Participated in the Virtual Robot Competition.

competition are utility-based mapping, victim detection, advanced mobility, and multirobot control. Utility-based mapping is the autonomous generation of maps from the fused data of multiple vehicles to be used by both robots and humans for exploration and marking victims. Victim detection is the automatic detection of victims from fused sensor data (image processing, acoustics, and others). Advanced mobility is accomplished by robust control algorithms capable of autonomous navigation in small spaces on nonflat flooring. Multirobot control is the ability to control multiple platforms with a single operator in realistic environments means that the robots have to be semiautonomous.

Originally a single scoring formula was used to evaluate the solutions associated with these issues. Simplified challenges were later introduced in 2009 to create more objective measures of performance. Each challenge was about a particular sub-problem with only one measure of performance and a corresponding automatic scoring tool. These challenges, namely mapping, navigation, and coordination over a mesh network, were used for qualification for the semifinals. Since 2011 the challenges have been combined again into a single mission. The goal is now defined in terms of an entirely automated scoring procedure. The scoring program is expected to allow for head-to-head competition between two competing teams. This also allows permanent installations of servers, each with its own world, which can be used for testing in preparation of the RoboCup. In this way, the teams can test their approach prior to the competition, hence providing a lower barrier of entry for new teams. Furthermore, the scoring procedure takes into account the individual subproblems solved in the comprehensive challenge so that the teams can assess their performance in all of the domains independently.

The main challenge for the teams is the control of a large team of robots (typically eight) by a single operator. This is state of the art; the only real comparison is the champion of the Magic competition (Olson et al. 2012), where 14 robots were controlled by two operators. The single operator has to use high-level commands (such as the areas to be searched, routes to be followed, and so on) but is also needed to verify observations as to 


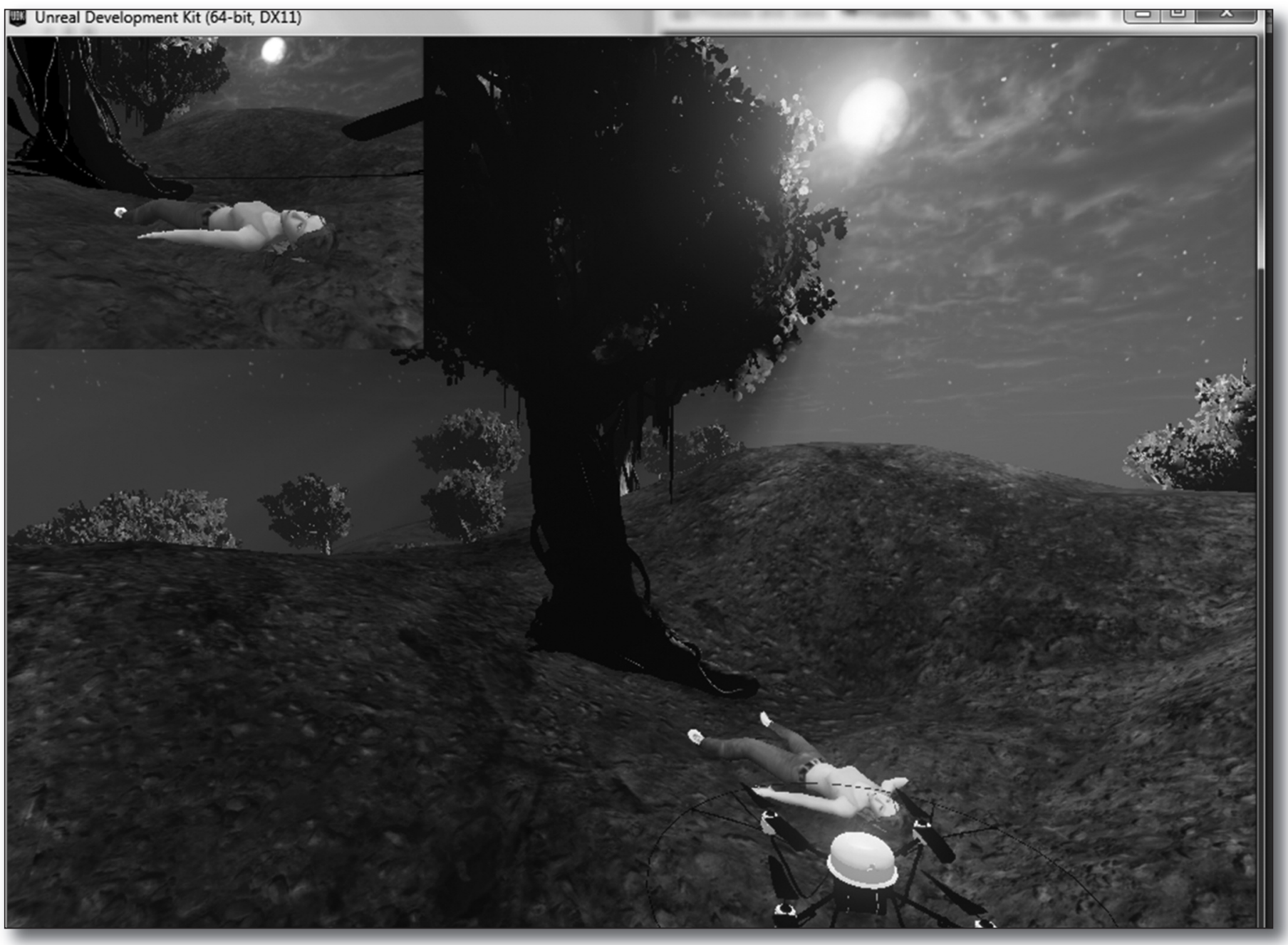

Figure 4. Outdoor Scenario for the Virtual Robot Competition in the Dutch Open Final 2012.

whether or not one of the robots has detected a victim (based on color or shape). Due to poor lighting and the number of occlusions, the conditions are generally not favorable for automatic victim detection, and manual conformation is always needed. The approach to a victim is quite critical (the robot should come within the communication range $[<1 \mathrm{~m}]$ but is not allowed to touch the body or any of the limbs). This means that the workload for the operator is quite high, providing an advantage for the teams that are able to automate the decision making within the robot team as far as possible and involve the operator only when needed.

The shared map generated during the competition has a central role in the coordination of such large robot teams. This is where the sensor information selected to be broadcast over often unreliable communication links is collected and registered. The registration process is asynchronous; some information may arrive at the base station even minutes after the actual observation. There is no guarantee that the operator has time to look at this information directly, which implies that the map within the user interface has to be interactive and should allow the operator to call back observations that were made at any point of interest (independent of when the observation was made and by which robot). At the same time the registration process should keep the map clean (no false positives or wrong associations), because it is the area where the coordination of the team behaviors is done.

In the final of the RoboCup 2012 competition the teams were able to find 12 of the 13 victims. The champion did this in 33 minutes (40 minutes were allowed). The competence level of the finalists is so high that next year the competition maps will have to be scaled up (both in size and difficulty). A possible way of increasing the difficulty of 
the competition is to include outdoor scenarios, as already demonstrated during the Dutch Open (see figure 4).

There are many publications related to this competition. ${ }^{3}$ The approach of the winning team in RoboCup 2012 will be published in the Lecture Notes on Artificial Intelligence series (Amigoni, Visser, and Tsushima 2013).

\section{Outlook}

The next competition will be in June 2013 in the Netherlands, followed in 2014 by a competition in Brazil. Colocating this competition with a demonstration of the progress of the teams participating in the DARPA Robotics Challenge is also intended. Information on the competition is available on several websites, software repositories, and fora. A good introduction to both leagues can be found on the wiki of the RoboCup organization. ${ }^{4}$

\section{Notes}

1. The current status of the common software framework for the RoboCup Rescue initiative is documented in the ROS wiki at www.ros.org/wiki/robocup_rescue.

2. See RMas-Bench-Rescue Multi-Agent Benchmarking by A. Kleiner, C. Dornhege, and A. Hertle, available online at kaspar.informatik.uni-freiburg.de/ rslb.

3. See www.robocuprescue.org/wiki/index.php?title=Publications_on_Virtual_Robots_and_USARSim.

4. See wiki.robocup.org/wiki/Main Page\#RoboCup_Rescue.

\section{References}

Amigoni, F.; Visser, A.; and Tsushima, M. 2013. RoboCup 2012 Rescue Simulation League Winners. In Robocup 2012: Robot Soccer World Cup XVI, Lecture Notes in Artificial Intelligence. Berlin: Springer.

Farinelli, A.; Rogers, A.; Petcu, A.; and Jennings, N. R. 2008. Decentralised Coordination of Low-Power Embedded Devices Using the Max-Sum Algorithm. In Proceedings of the Seventh International Conference on Autonomous Agents and Multiagent Systems (AAMAS 2008), 639-646. Richland, SC: International Foundation for Autonomous Agents and Multiagent Systems.

Ferreira, P.; Dos Santos, F.; Bazzan, A.; Epstein, D.; and Waskow, S. 2010. RoboCup Rescue as Multiagent Task Allocation Among Teams: Experiments with Task Interdependencies. Autonomous Agents and Multi-Agent Systems 20(3): 421-443.

Fitzpatrick, S., and Meetrens, L. 2003. Distributed Coordination through Anarchic Optimization. In Distributed Sensor Networks: A Multiagent Perspective, Volume 9, ed. V. Lesser, C. Ortiz Jr., and M. Tambe, 257-293. Dortrecht, the Netherlands: Kluwer.

Kitano, H., and Tadokoro, S. 2001. A Grand Challenge for Multiagent and Intelligent Systems. AI Magazine 22(1): 39-52.

Kitano, H.; Tadokoro, S.; Noda, I.; Matsubara, H.; Taka- hashi, T.; Shinjou, A.; and Shimada, S. 1999. RoboCup Rescue: Search and Rescue in Large-Scale Disasters as a Domain for Autonomous Agents Research. In Proceedings, IEEE 1999 Conference on Systems, Man, and Cybernetics (SMC-99). Piscataway, NJ: Institute for Electrical and Electronics Engineers.

Kleiner, A., and Dornhege, C. 2009. Operator-Assistive Mapping in Harsh Environments. In Proceedings, 2009 IEEE International Workshop on Safety, Security, and Rescue Robotics, 1-6. Piscataway, NJ: Institute for Electrical and Electronics Engineers.

Kleiner, A.; Brenner, M.; Bräuer, T.; Dornhege, C.; Göbelbecker, M.; Luber, M.; Prediger, J.; Stückler, J.; and Nebel, B. 2005. Successful Search and Rescue in Simulated Disaster Areas. In Robocup 2005: Robot Soccer World Cup IX, Lecture Notes in Computer Science Volume 4020, ed. A. Bredenfeld, A. Jacoff, I. Noda, and Y. Takahashi, 323-334. Berlin: Springer.

Kohlbrecher, S.; Meyer, J.; von Stryk, O.; and Klingauf, U. 2011. A Flexible and Scalable SLAM System with Full 3D Motion Estimation. In Proceedings, 2011 IEEE International Symposium on Safety, Security and Rescue Robotics. Piscataway, NJ: Institute for Electrical and Electronics Engineers.

Murphy, R. R.; Tadokoro, S.; Nardi, D.; Jacoff, A.; Fiorini, P.; Choset, H.; and Erkmen, A. M. 2008. Search and Rescue Robotics. In Springer Handbook of Robotics, ed. B. Siciliano and O. Khatib, chapter 50, 1151-1173. Berlin: Springer.

Nagatani, K.; Kiribayashi, S.; Okada, Y.; Tadokoro, S.; Nishimura, T.; Yoshida, T.; Koyanagi, E.; and Hada, Y. 2011. Redesign of Rescue Mobile Robot Quince - Toward Emergency Response to the Nuclear Accident at Fukushima Daiichi Nuclear Power Station on March 2011. In Proceedings, 2011 IEEE International Symposium on Safety, Security, and Rescue Robotics, 13-18. Piscataway, NJ: Institute for Electrical and Electronics Engineers.

Nair, R.; Ito, T.; Tambe, M.; and Marsella, S. 2002. Task Allocation in the RoboCup Rescue Simulation Domain: A Short Note. In RoboCup 2001: Robot Soccer World Cup V, Lecture Notes in Computer Science Volume 2377, ed. A. Birk, S. Coradeschi, S. Tadokoro, 751-754. Berlin: Springer.

Olson, E.; Strom, J.; Morton, R.; Richardson, A.; Ranganathan, P.; Goeddel, R.; Bulic, M.; Crossman, J.; Marinie, B. 2012. Progress Towards Multi-Robot Reconnaissance and the MAGIC 2010 Competition. Journal of Field Robotics 29(5): 762-792.

Pellenz, J., and Paulus, D. 2009. Stable Mapping Using a Hyper Particle Filter. In RoboCup 2009: Robot Soccer World Cup XIII, Lecture Notes in Computer Science Volume 5949, ed. J. Baltes, M. G. Lagoudakis, T. Naruse, and S. Shiry. 252-263. Berlin: Springer.

Ramchurn, S.; Farinelli, A.; Macarthur, K.; and Jennings, N. 2010. DecentralizedCoordination in RoboCup Rescue. The Computer Journal 53(9): 1447-1461.

Schurr, N., and Tambe, M. 2008. Using Multi-Agent Teams to Improve the Training of Incident Commanders. In Defence Industry Applications of Autonomous Agents and Multi-Agent Systems, ed. Michal Pechoucek, Simon G. Thompson, and Holger Voos, 151-166. Basel, Switzerland: Birkhäuser Verlag.

Tadokoro, S.; Kitano, H.; Takahashi, T.; Noda, I.; Matsub- 


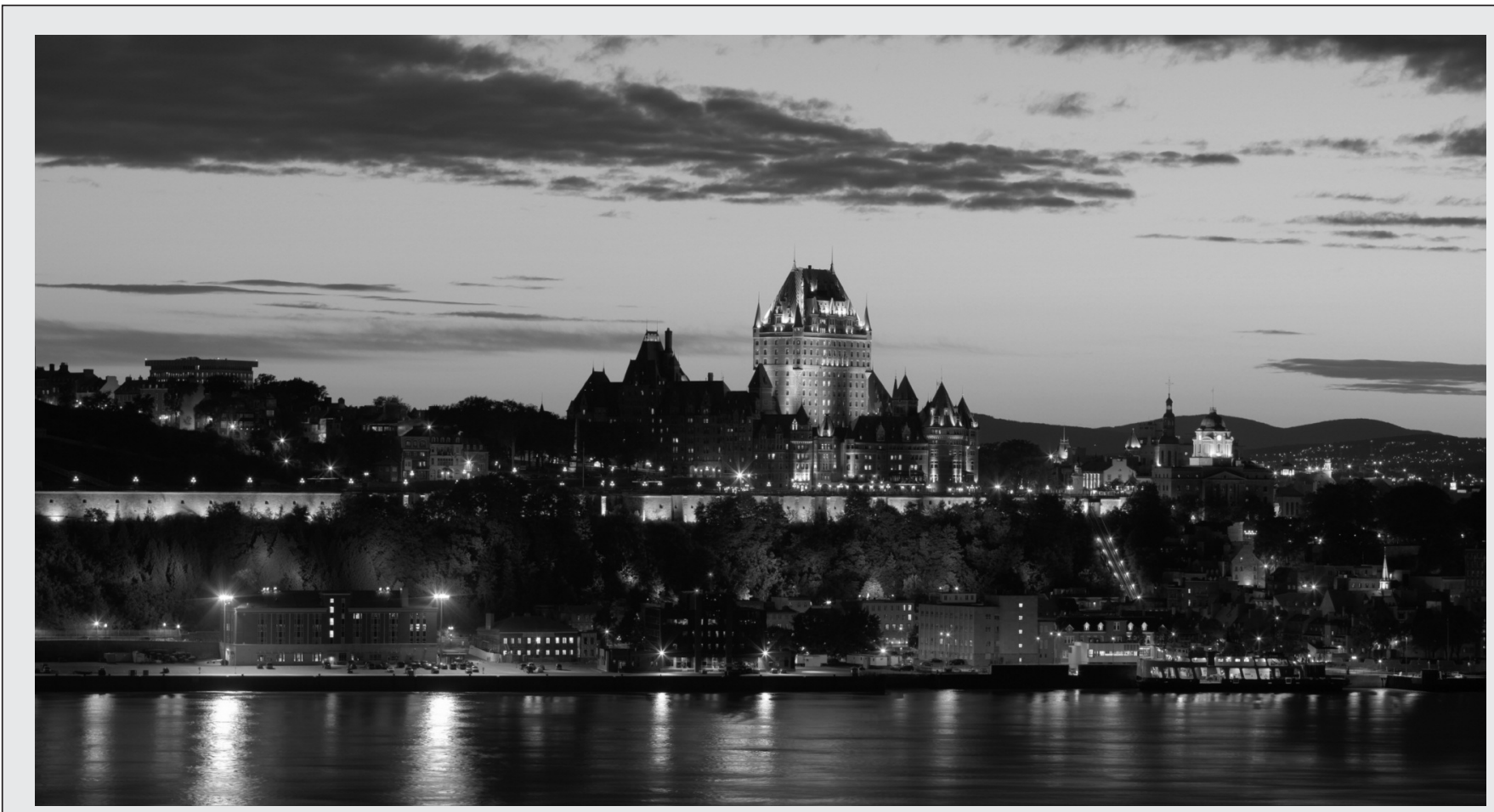

\section{AAAI to Colocate with Cognitive Science Society in 2014!}

AAAI is pleased to announce that it will colocate with the 2014 Cognitive Science Society Conference in picturesque Québec City, Québec, July 27-31, 2014. The conference will be held at the beautiful Centre des congrès de Québec, and attendees can stay at the adjacent Hilton Québec. More details will be available at the AAAI website soon!

ara, H.; Shinjou, A.; Koto, T.; Takeuchi, I.; Takahashi, H.; Matsuno, F.; Hatayama, M.; Nobe, J.; and Shimada, S. 2000. The RoboCup Rescue Project: A Robotic Approach to the Disaster Mitigation Problem. In Proceedings, IEEE International Conference on Robotics and Automation, 2000, volume 4, 4089-4094. Piscataway, NJ: Institute for Electrical and Electronics Engineers

Wirth, S., and Pellenz, J. 2007. Exploration Transform: A Stable Exploring Algorithm for Robots in Rescue Environments. In Proceedings of the 2007 IEEE International Workshop on Safety, Security, and Rescue Robotics, 1-5. Piscataway, NJ: Institute for Electrical and Electronics Engineers.

H. Levent Akin is a professor of computer engineering at Bogazici University, Turkey. He is a trustee of the RoboCup Federation and was the general chair of RoboCup 2011. He has more than 100 publications on AI and intelligent robotics.

Nobuhiro Ito is an associate professor of the faculty of information science at Aichi Institute of Technology, Japan. He is a member of the Executive Committee of the RoboCup Federation. His current research interests are protocols and algorithms for teamwork and contributions for real world by disaster simulations.

Adam Jacoff is a robotics research engineer in the Intelligent Systems Division of the National Institute of Stan- dards and Technology (NIST). His current efforts are focused toward developing standard test methods for emergency response robots to objectively evaluate robot performance and support development of advanced mobile robot capabilities. He is a trustee of the RoboCup Federation.

Alexander Kleiner is an associate professor of computer and information science at Linköping University, Sweden. He is a member of the Executive Committee of the RoboCup Federation. His research interests include (D)SLAM, exploration, and multirobot team coordination, and applying these techniques in the context of search and rescue.

Johannes Pellenz is the managing partner of V\&R Vision \& Robotics GmbH, Germany. The company develops solutions for imaging, mapping, and robotics. He is a member of the Executive Committee of the RoboCup Federation. His research interests include two-dimensional and three-dimensional SLAM, exploration, and standard software architectures for mobile robots.

Arnoud Visser is an assistant professor at University of Amsterdam. He is the chair of the Dutch RoboCup Committee. He is associate chair of RoboCup 2013 competitions and cochair of the RoboCup 2013 symposium. In addition, he is a member of the Executive Committee of the RoboCup Federation. His current research interest is focused on exploration with multirobot systems. 